\title{
Repeat Pregnancy after Prior Aortic Valve-in-Valve Replacement: A Cautionary Tale
}

\author{
Kelly Rasmussen, NP; Roxann Rokey, MD; Stacey C. Rolak MPH; Chuyang Zhong, MD; \\ John H. Braxton, MD, MBA; and Kazumasa Hashimoto, MD
}

\begin{abstract}
Transcatheter aortic valve replacement (TAVR) within a severely stenotic native aortic valve or previously placed surgical biologic aortic valve replacement (SAVR) is a rare occurrence in pregnant patients. The short- and long-term procedural outcomes for future pregnancies in these women or any woman of child bearing age who have received prior TAVR or TAVR in SAVR, are unknown. We describe the first result of a repeat pregnancy outcome in a woman with a history of prior TAVR in SAVR. Both maternal and fetal outcomes were favorable, but maternal cardiac complications observed in the third trimester emphasize our concerns regarding risk for cardiac complications in subsequent pregnancies in patients with a prior TAVR in SAVR. Despite the maternal complications that occurred during repeat pregnancy in this patient, a successful pregnancy outcome reaffirms our recommendation to utilize a multidisciplinary team for pregnancy management in patients with prior TAVR or TAVR in SAVR and to help in the management of any cardiac complications that may occur during or shortly after pregnancy.
\end{abstract}

Keywords: Chest pain; Heart valve prosthesis; Pregnancy complications; Transcatheter aortic valve replacement; Troponin I

$\mathrm{T}$ reatment of symptomatic, severely degenerated, biologic surgical aortic valve replacement (SAVR) using transcatheter aortic valve replacement (TAVR) has recently increased, particularly in the elderly. ${ }^{1-5}$ This procedure has not been routinely performed or clinically evaluated for women of reproductive age in either the pregnant or non-pregnant state. Previously, individual case reports have described five women who received either TAVR for a severely stenotic aortic valve or TAVR in SAVR for symptomatic stenosis during pregnancy ${ }^{6-10}$ Following this procedure, all women had uncomplicated post-operative recovery, uneventful pregnancies, and successfully delivered healthy infants. However, outcomes of repeat pregnancy in women with prior TAVR or TAVR in SAVR are unreported at this time, and concerns have been raised regarding subsequent pregnancies in these women. ${ }^{10-13}$ We describe the course of pregnancy, maternal, and fetal outcomes in a woman with prior TAVR in SAVR, and we reaffirm our recommendation to assemble a multidisciplinary team in the management of patients with prior TAVR or TAVR in SAVR who wish to conceive or who become pregnant.

\section{Case Description}

A 30-year-old gravida 3 para 1 woman sought prenatal care at our facility with an unplanned pregnancy at 7 weeks gestation. This patient was known to our obstetrics department from a recent previous viable pregnancy and successful delivery. With the previous pregnancy, she had presented in her late $20 \mathrm{~s}$ with symptomatic severe stenosis of a degenerative $27 \mathrm{~mm}$ SAVR Freestyle Stentless Bioprothesis (FSB) (Medtronic, Minneapolis, MN) that had been placed at the age of 16 for treatment of congenital aortic valve disease with severe aortic regurgitation. At that operation, the aorta and aortic valve annulus were not enlarged. The native aortic valve leaflets
Corresponding Author: Roxann Rokey, MD, Marshfield Clinic Research Institute, Clinical Research Center (GR3), 1000 North Oak Avenue, Marshfield, WI 54449, Tel: (7I5) 22I-6I2I, Email: rokey.roxann@marshfieldclinic.org
Received: January 17, 2020

Revised: June 2, 2020

Accepted: June 23, 2020 
were excised and the annulus left intact to anchor the FSB. The left and right coronary ostia were removed from the aorta with a patch of autologous aortic tissue and the FSB was rotated in its anatomic position. Anastomosis of the left ventricle was performed by connecting the proximal segment of the FSB device at the native aortic annulus. An appropriate opening was made in the left and right coronary sinuses with subsequent anastomosis of the left then right coronary artery. Lastly, the aorta was reconstructed by connecting the distal part of the FSB to the ascending aorta.

During the prior pregnancy, a TAVR in SAVR procedure using a $26 \mathrm{~mm}$ CoreValve Evolut R valve (Medtronic, Minneapolis, $\mathrm{MN}$ ) was performed at 12-weeks gestation with resolution of aortic stenosis and symptoms. The remainder of the pregnancy was uneventful, and she delivered a healthy male infant by Cesarean delivery (due to arrest of dilatation). Post-partum, she remained asymptomatic from a cardiac perspective, and the new TAVR in SAVR was stable. One day post TAVR in SAVR hemodynamics were considered in the normal range and were similar those obtained one month post-partum. One day post TAVR in SAVR procedure valve hemodynamics showed a Doppler velocity index $(\mathrm{DVI})>3.0$, estimated valve area of $1.2 \mathrm{~cm}^{2}$, peak aortic valve velocity of $2.9 \mathrm{~m} / \mathrm{sec}$, peak gradient of $33 \mathrm{mmHg}$, and mean gradient of $28 \mathrm{mmHg}$. One month post-partum, the hemodynamics showed a DVI of $>3.0$, an estimated valve area of $1.26 \mathrm{~cm}^{2}$, peak velocity of 2.2 $\mathrm{m} / \mathrm{s}$, with peak and mean gradient of $20 \mathrm{mmHg}$ and 12 $\mathrm{mmHg}$, respectively. Normal values for this valve at our laboratory is DVI $>3.0$, estimated valve area $\geq 1.2 \mathrm{~cm}^{2}$, peak velocity $<3 \mathrm{~m} / \mathrm{sec}$, with peak and mean gradient of $\leq 36$ $\mathrm{mmHg}$ and $<20 \mathrm{mmHg}$, respectively. She was counseled for appropriate contraception and advised to delay future pregnancies until the full impact of the recent TAVR in SAVR in the short- and long-term was fully evaluated. ${ }^{10}$

Seven months later, at the time of presentation with current pregnancy, the patient had no cardiac symptoms or noncardiac symptoms other than minor vaginal spotting prior to the appointment. Repeat echocardiogram showed stability of the TAVR bioprosthesis hemodynamics with no significant elevation of gradient or perivalvular regurgitation compared to baseline post-procedure values. Our multidisciplinary, high-risk pregnancy team re-established for management of pregnancy, labor, and delivery, and the patient was counseled to continue her aspirin regimen $(81 \mathrm{mg} /$ day). Although offered monthly follow-up visits by maternal/fetal medicine and cardiology departments, due to travel constraints, she missed several prenatal appointments. Serial echocardiograms continued to confirm stable TAVR valve hemodynamics (Table 1). No significant perivalvular regurgitation developed.

She and her fetus did well throughout the pregnancy until 37-weeks gestation. Fetal anatomy survey and fetal echocardiography were normal in the second trimester. Follow-up obstetric ultrasound examination was performed periodically every 4 to 6 weeks which demonstrated normal fetal growth. Biophysical profile test was reassuring (8/8) at 32 weeks, 36 weeks, and 37 weeks. At 37 weeks gestation, she developed substernal chest pain radiating to the left arm lasting for 7 hours. The patient presented to the emergency department for evaluation via private vehicle. She entered the facility in a wheelchair with family and was immediately taken back to an ER room. This was her first episode of chest pain. Her symptoms were described as $2 / 10$ continuous mild to moderate heavy central radiating to the left shoulder, and worse with inspiration. There was no shortness of breath, worsening of chest discomfort with activity throughout the day before coming to the ER, no diaphoresis, palpitations, syncope or near syncope. Initial blood pressure was $118 / 59$ $\mathrm{mmHg}$ with a physician recorded heart rate of 102 beats per minute and oxygen saturation of $98 \%$ on room air.

Her cardiac examination was unchanged compared to prior evaluations, except for tachycardia with a heart rate of 102 beats per minute. Her chest discomfort resolved spontaneously in the ER with no medications being given. Electrocardiogram (EKG) obtained 10 minutes after arrival to the ER during her

Table 1. Serial Doppler Echocardiogram of TAVR in SAVR hemodynamics prior to repeat pregnancy and during current pregnancy.

\begin{tabular}{|c|c|c|c|c|c|c|c|}
\hline & \multirow{2}{*}{$\begin{array}{c}5 \text { months } \\
\text { prior to } \\
\text { pregnancy }\end{array}$} & \multicolumn{6}{|c|}{ Weeks of gestation } \\
\hline & & 4 & 13 & 18 & 22 & 30 & 37 \\
\hline DVI & $>0.30$ & $>0.30$ & $>0.30$ & $>0.30$ & $>0.30$ & $>0.30$ & $>0.30$ \\
\hline EOA & 1.12 & 1.22 & 1.34 & 1.92 & 1.28 & 1.21 & 1.39 \\
\hline Peak gradient $(\mathrm{mmHg})$ & 25 & 31 & 33 & 19 & 36 & 35 & 29 \\
\hline Mean gradient $(\mathrm{mmHg})$ & 15 & 17 & 19 & 11 & 21 & 14 & 17 \\
\hline $\operatorname{Vmax}(\mathrm{m} / \mathrm{sec})$ & 2.52 & 2.78 & 2.87 & 2.15 & 2.99 & 2.95 & 2.69 \\
\hline
\end{tabular}

Abbreviations: DVI=Doppler velocity index; EOA=Estimated orifice area; Vmax=maximal Doppler velocity; weeks=approximate gestational age 

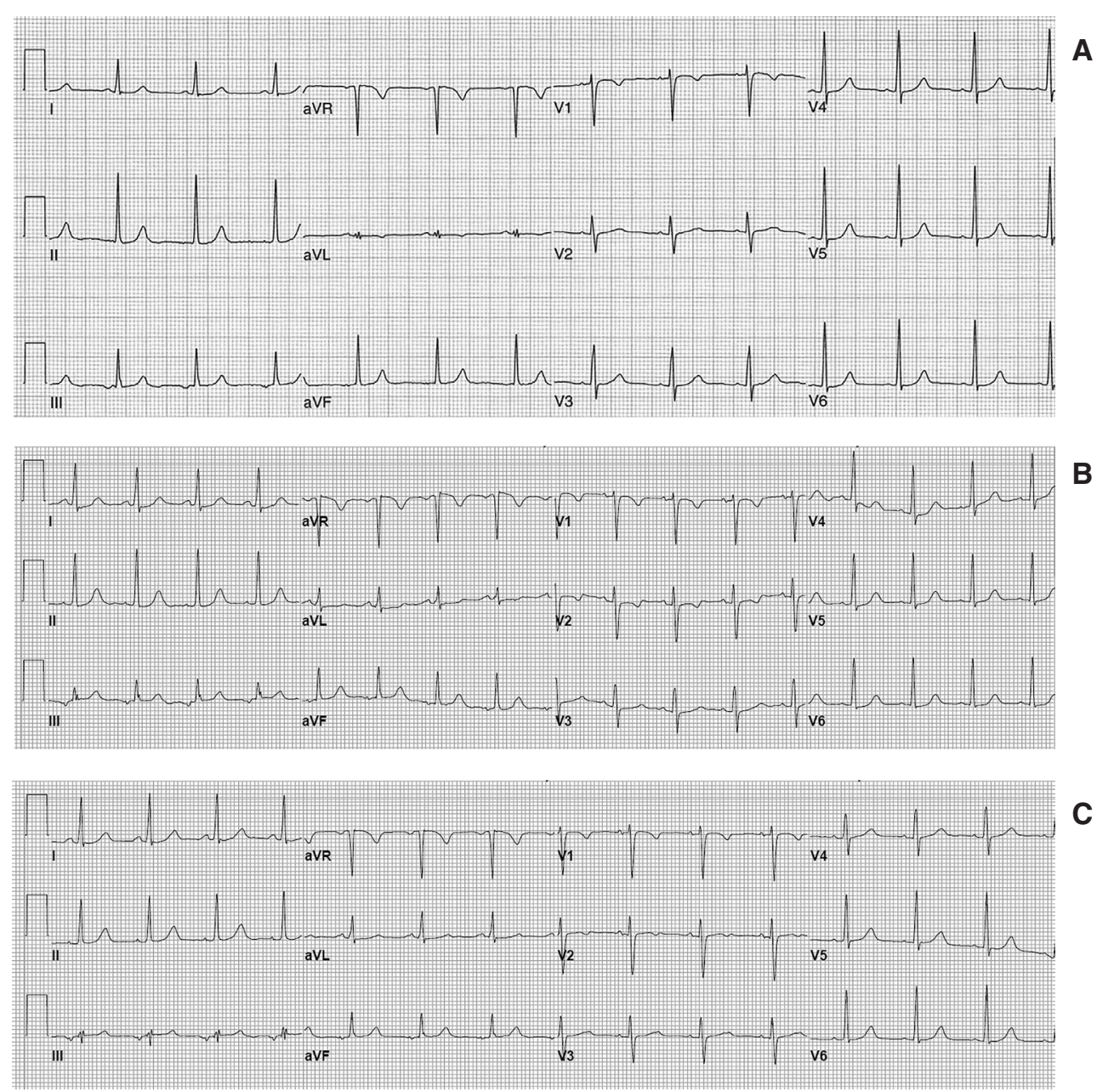

Figure 1. Comparison of electrocardiograms one week prior to complaints of chest pain (A), during emergency room visit with Troponin I (TNI) elevation (B), and one day after admission (C). The verified recorded heart rates on Figure A-C were 78 bpm, 102 bpm and 87 bpm, respectively. No evidence of ST elevation myocardial infarction is present. Although a new isolated $T$ wave in V2 is present in both emergency room and hospital electrocardiogram, this is not diagnostic of ischemia. chest discomfort showed no definitive signs of ischemia compared to prior EKGs (Figure 1). In the emergency department, Troponin I (TNI) level was initially normal at 39 $\mathrm{ng} / \mathrm{L}$ (normal range 0 to $45 \mathrm{ng} / \mathrm{L}$ ); $45 \mathrm{ng} / \mathrm{mL}$ is 99 th percentile upper reference limit normal value for women within our system). Serial TNI levels increased beyond normal at two hours with value of $68 \mathrm{ng} / \mathrm{mL}$, peaked at $447 \mathrm{ng} / \mathrm{mL}$ at 12 hours, and trended down thereafter.

Extensive evaluations for causes of chest pain with concurrent elevated TNI levels ensued. The patient did not have a history of recurrent miscarriages or venous/arterial thromboembolism that would increase her risk for pulmonary embolism or myocardial infarction. Bilateral lower extremity venous duplex was negative for deep venous thrombosis. Review of prior gated computed tomography (CT) angiogram of the chest done 20 months earlier showed calcification of the SAVR FBS root but no obstruction of the reimplanted coronary arteries, no obstructive calcified or non-calcified coronary plaques, and normal aortic size (Figure 2). Transthoracic echocardiogram showed normal cardiac function and wall motion, and no aortic dissection. TAVR leaflets were clear of mobile masses and were not significantly thickened. There was no significant change in peak or mean gradients from baseline (Table 1) or degree of valvular regurgitation that would be concerning for progressive obstruction of the valve leaflets. All the patient's values appeared to be well within the normal prosthetic values reported in our system. Fetal growth was normal, and fetal status remained reassuring. Fetal growth was normal at 36 weeks and fetal status showed a biophysical profile score of $8 / 8$ at 37 weeks.

Based on the patient's recent past history of TAVR in SAVR FSB with symptoms suggesting cardiac etiology and characteristic TNI elevation, it was thought the patient had non ST elevation myocardial infarction (NSTEMI) type $2 .{ }^{14}$ Members of the multidisciplinary group were contacted to determine the next steps in patient management, and multiple etiologies for NSTEMI in pregnancy were discussed. Because she was clinically and hemodynamically stable with no evidence of STEMI, cardiac catheterization was not pursued. ${ }^{15,16}$ A CT angiogram was not pursued at initial presentation/evaluation given the patient's young age, prior 


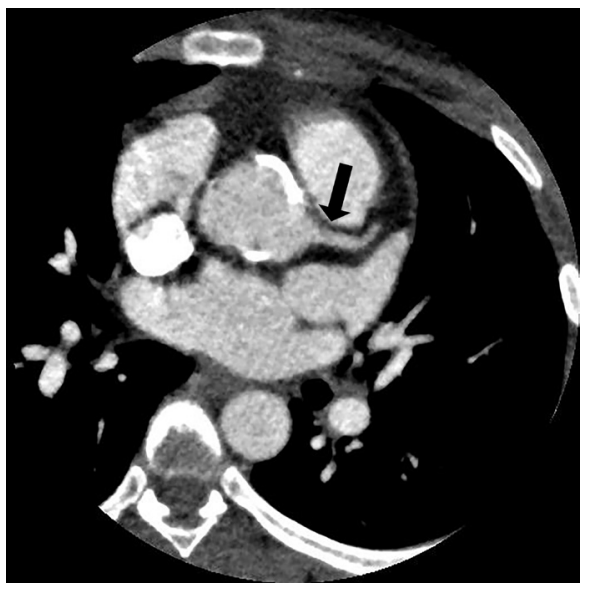

A

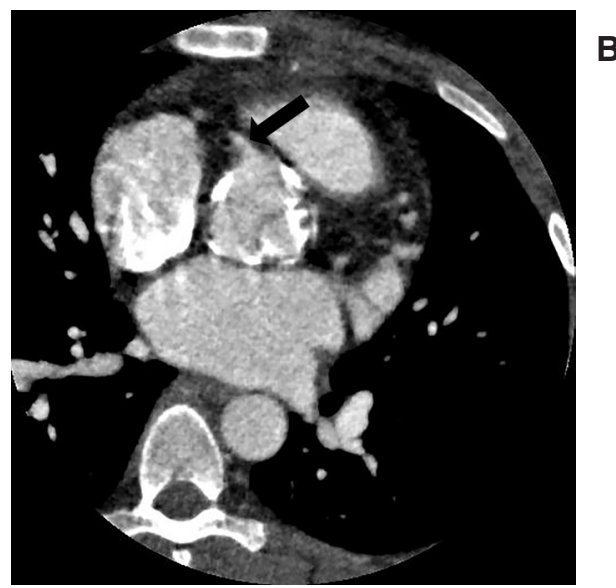

Figure 2. Gated computed tomography of the chest 20 months earlier during prior pregnancy showing no plaque obstruction of the left main/proximal left anterior descending artery coronary artery ( $\mathrm{A}$, black arrow) or proximal right coronary artery (B, black arrow). Likewise, no obstructive disease was seen in the mid, distal, or branch vessels of the remaining coronary artery territories. known CT angiogram results, current pregnant state with concerns of potential fetal and maternal radiation exposure as well as potential long term fetal effects from contrast. There was low concern for obstructive native atherosclerotic calcific coronary artery disease based on prior recent CT angiogram findings and since the patient was clinically and hemodynamically stable, without recurrent chest pain and was being treated for NSTEMI, it was felt that results including localized coronary hematoma, dissection or thrombus would not alter her current initial management. She was continued on aspirin at $81 \mathrm{mg} /$ day, placed on intravenous heparin, and given a beta-blocker (metoprolol $6.25 \mathrm{mg}$ twice a day orally) with adjustment for heart rate as needed. The dose of heparin was based on system wide nomogram for acute coronary syndrome. A bolus of 60 units $/ \mathrm{kg}$ followed by a continuous intravenous infusion with adjustments to achieve partial thromboplastin time (PTT) of 50-70 seconds. Values were checked 4 hours after adjustments or otherwise every morning. Since repeat Cesarean delivery was planned once the patient was stabilized, P2Y12 inhibitors were not initiated. The patient had no recurrent chest pain for the remainder of her hospital stay. At 38 weeks gestation, heparin was discontinued, and she had a repeat Cesarean delivery under general anesthesia with delivery of a healthy male infant weighing 3,785 g. Transesophageal echocardiogram (TEE) with 3D imaging performed at the time of delivery confirmed there were no obvious valvular masses on the TAVR bioprosthesis leaflets or abnormalities located above or below the aortic position. The leaflets appeared to be thin and pliable (Figure 3 ). No masses were seen in the coronary sinuses or any obvious abnormalities noted above or below the aortic positon. The LV and RV function were normal. No regional wall motion abnormalities were seen. No pericardial effusion was present. A follow-up gated chest CT 1 day after delivery confirmed no aortic dissection or pulmonary emboli. The origins of the coronary arteries and visualized epicardial vessels appeared to be normal (Figure 4). It was felt by the team that since the patient was clinically stable with no
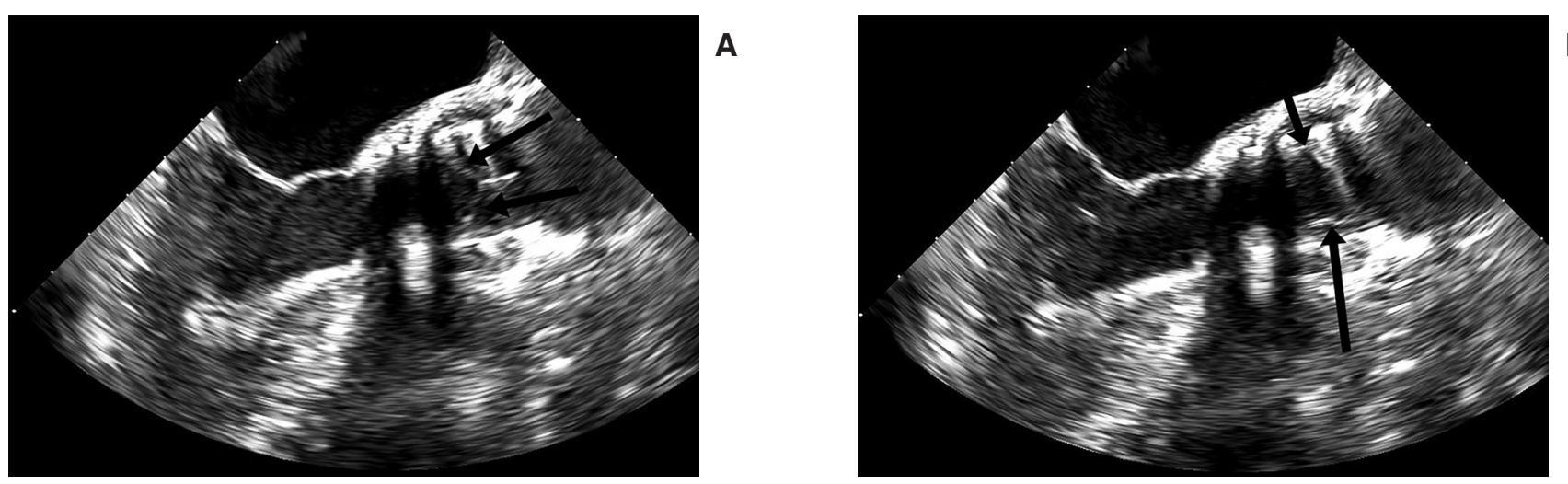

Figure 3. TEE done during cesarean section at the time of the second delivery. Images show the long axis of the aortic TAVR in SAVR aortic valve leaflets and root. The frame of the TAVR device is echogenic and reflective (large black arrows). The TAVR valve leaflets are thin and demonstrate pliability as seen in diastole just before the leaflets open (A, narrow black arrows) and then in full systole ( $B$, narrow black arrows). 


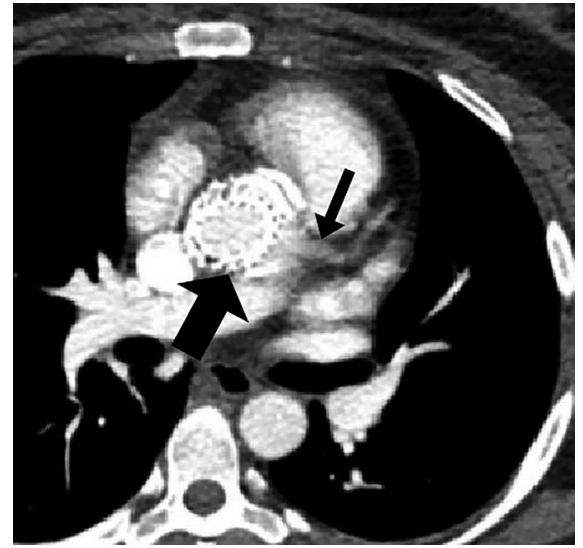

A

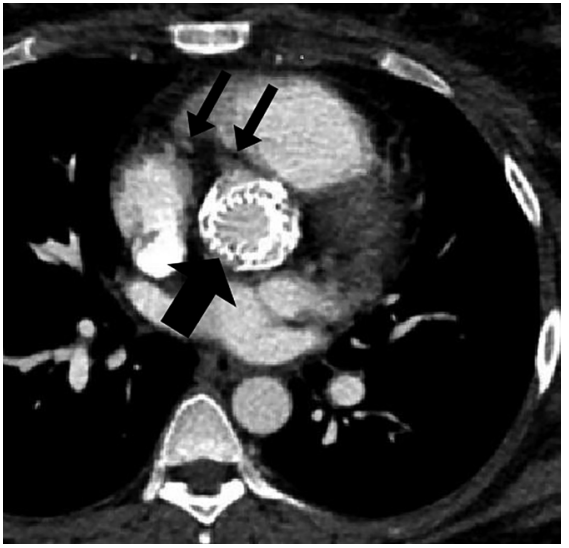

B

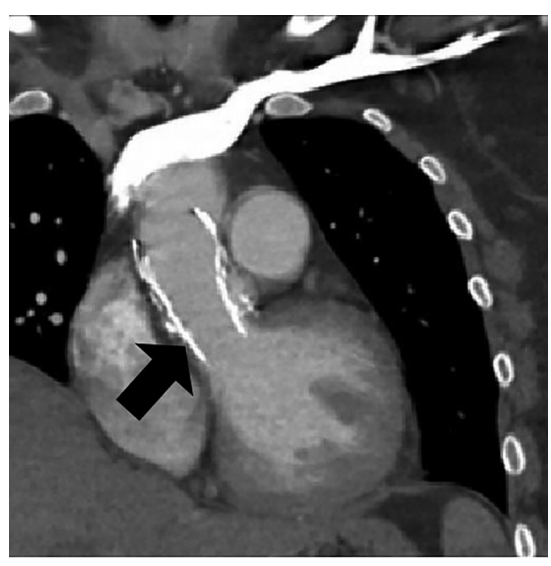

C

Figure 4. Repeat gated computed tomography of the chest 1 day after delivery this current pregnancy. A TAVR in SAVR is seen in the location of the aortic root and ascending aorta both in the horizontal view and the coronal views ( $A-C$, large black arrows). There was no obvious thrombus, localized hematoma or dissection seen in the left main/proximal left anterior descending artery coronary artery ( $\mathrm{A}$, narrow black arrow), or proximal right coronary artery ( $\mathrm{B}$, narrow black arrows). Likewise, no obstructive disease was seen in the mid, distal, or branch vessels of the remaining coronary artery territories. The coronal view is windowed to show the frame of the TAVR valve and no obvious abnormalities are seen (C, large black arrow).

obvious abnormalities identified by TEE with 3D imaging or on CT scan that no additional radiologic or invasive procedures for risk stratification were necessary.

Following delivery, a therapeutic dose of low-molecular weight heparin was administered for 6 weeks along with discharge medications of beta-blockers and aspirin. Clopidogrel was declined by the patient because of breast feeding. The patient remained asymptomatic from a cardiac perspective at a follow-up visit. Contraceptive measures and initiation of clopidogrel after systemic anticoagulation was recommended. Discussions on multiple visits regarding delay of any future pregnancies were again made to the patient. She agreed to appropriate contraceptive measures but continued to decline P2Y12 inhibitors until after she stopped breastfeeding. In three follow-up appointments over 7 weeks with the cardiology and obstetrics departments, she remained asymptomatic. A routine follow-up echocardiogram ordered 2 months after delivery showed preserved LV and RV function with no wall motion abnormalities but increasing aortic valve gradients (a peak gradient of $44 \mathrm{mmHg}$, a mean gradient of 28 $\mathrm{mmHg}$ ) compared to the last study done at 37-weeks gestation. The patient was called with results and strongly urged to follow-up at the clinic to discuss these findings of possible TAVR valve dysfunction and further management options. She declined a follow-up visit, citing asymptomatic state, health insurance constraints, and plans of an impending move. She subsequently moved to a different part of the country and has been lost to follow-up at our clinic.

\section{Discussion}

The use of TAVR for symptomatic severe aortic stenosis or TAVR in SAVR (also known as aortic valve-in-valve) is a recent promising procedure that provides a less-invasive surgical option for those with symptomatic severe native or surgically implanted biologic aortic valve stenosis. ${ }^{1-3}$ This TAVR technique is primarily used in elderly patients and rarely in women of child-bearing age..$^{4,5,11-13}$ As of this writing, there have only been five reports of young women receiving TAVR or TAVR in SAVR while pregnant, one with severe native aortic valve stenosis and four with TAVR in SAVR. ${ }^{6-10}$ It is unknown how women with a previous TAVR of severe aortic stenosis or TAVR in SAVR will tolerate subsequent pregnancies, due to lack of publications regarding short- and long-term maternal and fetal outcomes of repeat pregnancy after TAVR. Our case represents the first report of subsequent pregnancy outcome in a woman with prior TAVR in SAVR. Although our patient did well for the majority of her pregnancy, she developed acute coronary syndrome with NSTEMI type 2 late in the third trimester, but she ultimately fared well without further complications and delivered a healthy infant.

Bioprosthetic aortic valve replacement is recommended for severe aortic stenosis in women wishing to conceive. ${ }^{17}$ However, when placed in young patients, the valve deteriorates rapidly, with a prospect of re-intervention within one to two decades. ${ }^{17-22}$ In women with well-functioning biologic SAVR valves implanted prior to conception, it is rare for significant deterioration to occur during pregnancy, but this situation has been reported and may require intervention with TAVR in SAVR. ${ }^{9,23}$

With TAVR in SAVR, two prosthetic biologic valves are now implanted, and there is evidence in the elderly that this biologic valve-in-valve combination may experience more rapid deterioration and/or valve leaflet thrombosis when compared 
to those who have received a lone TAVR device. ${ }^{24}$ For younger patients in the non-pregnant state, the longer term efficacy of TAVR or TAVR in SAVR is not verified in terms of valve deterioration or thrombosis. ${ }^{10-13}$ Even more problematic are these unproven variables in women who become pregnant or who desire subsequent pregnancies. ${ }^{10}$

Because normal pregnancy is inherently a prothrombotic state, there may be an increased risk of valve thrombosis or thrombus formation above or below the TAVR in SAVR, since the device is smaller than the original bioprosthesis and can potentially serve as a nidus for thrombus formation during pregnancy. ${ }^{25}$ Furthermore, patients with a TAVR in SAVR could, in theory, experience calcific embolization due to the calcification present in a deteriorated FSB that is addressed via the TAVR in SAVR procedure. , $^{3,26-31}$

Myocardial infarction during pregnancy is an uncommon event with multiple possible etiologies for the condition, which may occur in the presence of normal or atherosclerotic coronary arteries. ${ }^{15,16,32-34}$ Possible etiologies for myocardial infarction include coronary atherosclerosis with or without associated thrombus, coronary artery dissection, localized hematoma, and coronary artery spasm. ${ }^{15,16,32-34}$ NSTEMI may occur in approximately $25 \%-30 \%$ of pregnant patients who experience myocardial infarction, and our patient had typical chest pain and TNI elevation consistent with NSTEMI. ${ }^{16,32}$ TNI elevation can occur as a result of hypertensive complications in pregnancy, including pre-eclampsia, but was not present in our patient. ${ }^{15,16,32,33}$ Obstructive coronary calcification can also occur, but prior gated CT angiogram done 17 months earlier before her initial TAVR in SAVR showed no obstructive coronary calcification or plaque and was unlikely to occur. ${ }^{15,16,32}$ Other potential causes of TNI elevation such as heart failure, aortic dissection, pulmonary embolism, and renal failure as a cause for the TNI elevation were not identified. We did not suspect valve thrombosis, since the aortic valve gradients were not significantly increased. However, at the time of presentation, we could not exclude the possibility that a valvular mass had embolized diffusely at the time of chest pain development, though no masses on the valve itself were revealed during imaging. It is possible that a prior coronary artery spasm, hematoma, or localized dissection could have occurred, but given the stability of the patient and lack of STEMI, we elected not to proceed with cardiac catheterization due to the inherent significant risks of this procedure during pregnancy. ${ }^{15,16,32}$ Because our patient had prior TAVR in SAVR, two possible new additional etiologies as a cause of NSTEMI related to the device could also be postulated, including embolization from micro thrombus formation in proximity of the device or calcific embolization within a deteriorated SAVR FSB.

Overt valve leaflet thrombosis was not suspected in our patient, since valve hemodynamics showed no significant increase in gradients throughout pregnancy. Aortic valve leaflet thrombosis of a TAVR in SAVR device has been reported in up to $7 \%$ of elderly patients and usually occurs within 3-6 months of implantation. ${ }^{24,35}$ This condition may be clinically silent and is less likely to be present if the patient is on oral anticoagulation prior to diagnosis. ${ }^{24}$ Additionally, thrombosis is seen more frequently in patients with TAVR in SAVR compared to patients receiving TAVR for improvement of native aortic valve stenosis, and it is more likely if the SAVR device is a stented biologic valve, or if a TAVR valve is deployed with a balloon expandable device. ${ }^{35}$ Postulated mechanisms for TAVR in SAVR thrombosis include flow stasis or increased blood resistance by the degenerated SAVR valve. ${ }^{35}$ There are no established guidelines regarding initiation and duration of oral anticoagulants in patients who have TAVR in SAVR, since the risk of valve thrombosis is low. ${ }^{17,18}$ Similarly the duration and number of antiplatelet agents following TAVR in SAVR has not been standardized. However, for patients with valve-in-valve thrombosis, systemic anticoagulation appears to be effective in substantially reducing gradients and rarely requires surgery. ${ }^{35}$ Even though overt TAVR in SAVR valve thrombosis was not detected in our patient, we suspect that the hypercoagulable state of pregnancy, in addition to the presence of TAVR in SAVR device and degenerated FSB, played a role in this patient's NSTEMI.

Theoretically in patients with TAVR in SAVR, there is a possibility of calcific embolization, particularly in severely calcified FSB. Calcific embolization to coronary arteries occurred in case reports of patients with native calcific aortic valves and may result in STEMI or be subclinical with diffuse distribution and no apparent clinical presentation. ${ }^{28-31}$ However, spontaneous calcium embolization of a TAVR in a severely degenerated SAVR or SAVR FSB during pregnancy or in nonpregnant state has not been previously reported, though we note that our patient received a prior TAVR in SAVR procedure to improve cardiac function in a severely stenotic calcified FSB. $^{10}$

Post-partum, the patient remained asymptomatic without chest pain or shortness of breath. Echocardiogram done 2 months after delivery showed findings concerning for possible TAVR valve dysfunction, with increasing aortic valve gradients. Although urged to follow-up regarding these findings, she declined. Because she moved from the region, we were unable to complete further evaluation of these findings or offer potential treatments. We cannot directly attribute increasing valve gradients to her recent pregnancy. Nevertheless, these findings amply reinforce our continued recommendations to urge an abundance of caution for repeat pregnancy in women with prior TAVR in SAVR until more short- and long-term results of the procedure are known.

\section{Conclusion}

This is the first case report of a repeat pregnancy and shortterm outcome in a woman with prior TAVR in SAVR. Because 
TAVR in SAVR is a relatively new procedure and infrequently performed on women of childbearing age, the short- and longterm consequences of this procedure and impact on repeat pregnancy outcome is not yet known. Our patient had complications of NSTEMI late in the third trimester. There is ample literature discussing possible etiologies of myocardial infarction in pregnancy. ${ }^{15,16,32}$ We postulate two new additional etiologies for myocardial infarction during pregnancy in women with prior TAVR in SAVR, including a hypercoagulable state associated with pregnancy to act as a nidus of thrombus formation in or near the TAVR in SAVR device, or coronary artery calcification embolization in degenerated TAVR in SAVR valves or root. Thrombus formation and calcification in such cases could potentially be identified by TEE with $3 \mathrm{D}$ imaging or 4D CT imaging with thin slice acquisition.

Although pregnancy outcomes in this patient cannot be extrapolated to all women of childbearing age with prior TAVR of severe native aortic valve stenosis or TAVR in SAVR, we still urge an abundance of caution for repeat pregnancy in these patients until more short- and long-term results of the procedure are known, particularly for TAVR in SAVR. For women with this procedural history who want to conceive, we recommend convening a high-risk pregnancy team with experience in management of high-risk cardiac patients to discuss treatment options and management. ${ }^{10,17}$

\section{Acknowledgements}

The authors acknowledge the Marshfield Clinic Research Institute's Scientific Writing and Publication division for manuscript editing and submission.

\section{References}

1. Webb JG, Mack MJ, White JM, et al. Transcatheter aortic valve implantation within degenerated aortic surgical bioprostheses: PARTNER 2 valve-in-valve registry. J Am Coll Cardiol. 2017;69(18):2253-2262.

2. Pibarot P, Simonato M, Barbanti M, et al. Impact of preexisting prosthesis-patient mismatch on survival following aortic valve-in-valve procedures. JACC Cardiovasc Interv. 2018;11(2):133-141.

3. Carabello BA. Valve-in-Valve TA VR: Insights into the pathophysiology of aortic stenosis. J Am Coll Cardiol. 2017;69(18):2263-2265.

4. Isaacs AJ, Shuhaiber J, Salemi A, Isom OW, Sedrakyan A. National trends in utilization and in-hospital outcomes of mechanical versus bioprosthetic aortic valve replacements. J Thorac Cardiovasc Surg. 2015;149(5):1262-1269.e3.

5. Messenger JC. Trends in United States TAVR Practice. An update on where TAVR is going as the technology matures in the United States. Cardiac Interventions Today 2018; 12(2): 46-50. Available at: https://citoday.com/2018/04/trends-inunited-states-tavr-practice. Last accessed November 24, 2020.

6. Gandhi S, Ganame J, Whitlock R, Chu V, Natarajan MK, Velianou JL. Double Trouble: A Case of Valvular Disease in Pregnancy. Circulation. 2016;133(22):2206-2211.

7. Herbert KA, Sheppard SM. Not your typical dyspnea of pregnancy: A case report of transcatheter valve-in-valve replacement during pregnancy. A A Practice. 2019;12(6):202204.
8. Hodson R, Kirker E, Swanson J, Walsh C, Korngold EC, Ramelli S. Transcatheter aortic valve replacement during pregnancy. Circ Cardiovasc Interv. 2016;9(10):e004006.

9. Berry N, Sawlani N, Economy K, et al. Transcatheter aortic valve replacement for bioprosthetic aortic stenosis in pregnancy. JACC Cardiovasc Interv. 2018;11(19):e161-e162.

10. Zhong C, Rokey R, Rolak S, Mesa J. Pregnancy and transcatheter aortic valve replacement in a severely stenotic Freestyle full aortic root stentless bioprosthesis. Catheter Cardiovasc Interv. 2020 May 1;95(6):1225-1229.

11. Sedrakyan A, Dhruva SS, Shuhaiber J. Transcatheter aortic valve replacement in younger individuals. JAMA Intern Med. 2017;177(2):159-160.

12. Sedrakyan A, Dhruva SS, Sun T, Mao J, Gaudino MFL, Redberg RF. Trends in use of transcatheter aortic valve replacement by age. JAMA. 2018;320(6):598-600.

13. Mazine A, Ouzounian M. Aortic valve replacement in young and middle-aged adults: looking beyond the tree that hides the forest. Ann Transl Med. 2017;5(4):92.

14. Thygesen K, Alpert JS, Jaffe AS, et al; Writing Group on the Joint ESC/ACCF/AHA/WHF Task Force for the Universal Definition of Myocardial Infarction; ESC Committee for Practice Guidelines (CPG). Third universal definition of myocardial infarction. Eur Heart J. 2012;33(20):2551-2567.

15. Roth A, Elkayam U. Acute myocardial infarction associated with pregnancy. J Am Coll Cardiol. 2008;52(3):171-180.

16. Elkayam U, Jalnapurkar S, Barakkat MN, et al. Pregnancyassociated acute myocardial infarction: a review of contemporary experience in 150 cases between 2006 and 2011. Circulation. 2014;129(16):1695-1702.

17. Nishimura RA, Otto CM, Bonow RO, et al; ACC/AHA Task Force Members. 2014 AHA/ACC Guideline for the Management of Patients With Valvular Heart Disease: executive summary: a report of the American College of Cardiology/American Heart Association Task Force on Practice Guidelines. Circulation. 2014;129(23):2440-2492.

18. Nishimura RA, Otto CM, Bonow RO, et al. 2017 AHA/ACC Focused Update of the 2014 AHA/ACC Guideline for the Management of Patients With Valvular Heart Disease: A Report of the American College of Cardiology/American Heart Association Task Force on Clinical Practice Guidelines. Circulation. 2017;135(25):e1159-e1195.

19. Sanaani A, Yandrapalli S, Harburger JM. Antithrombotic management of patients with prosthetic heart valves. Cardiol Rev. 2018;26(4):177-186.

20. Hammermeister K, Sethi GK, Henderson WG, Grover FL, Oprian C, Rahimtoola SH. Outcomes 15 years after valve replacement with a mechanical versus a bioprosthetic valve: final report of the Veterans Affairs randomized trial. J Am Coll Cardiol. 2000;36(4):1152-1158.

21. Rahimtoola SH. Choice of prosthetic heart valve in adults an update. J Am Coll Cardiol. 2010;55(22):2413-2426.

22. Badhwar V, Ofenloch JC, Rovin JD, van Gelder HM, Jacobs JP. Noninferiority of closely monitored mechanical valves to bioprostheses overshadowed by early mortality benefit in younger patients. Ann Thorac Surg. 2012;93(3):748-753.

23. El SF, Hassan W, Latroche B, et al. Pregnancy has no effect on the rate of structural deterioration of bioprosthetic valves: long-term 18-year follow up results. J Heart Valve Dis. 2005;14(4):481-485.

24. Jose J, Sulimov DS, El-Mawardy M, et al. Clinical bioprosthetic heart valve thrombosis after transcatheter aortic valve replacement: Incidence, characteristics, and treatment outcomes. JACC Cardiovasc Interv. 2017;10(7):686-697. 
25. Hellgren M. Hemostasis during normal pregnancy and puerperium. Semin Thromb Hemost. 2003;29(2):125-130.

26. Bach DS, Kon ND. Long-term clinical outcomes 15 years after aortic valve replacement with the Freestyle stentless aortic bioprosthesis. Ann Thorac Surg. 2014;97(2):544-551.

27. Amabile N, Bical OM, Azmoun A, Ramadan R, Nottin R, Deleuze PH. Long-term results of Freestyle stentless bioprosthesis in the aortic position: A single-center prospective cohort of 500 patients. J Thorac Cardiovasc Surg. 2014;148(5):1903-1911.

28. Vasconcellos AP, Korr KS. Spontaneous calcific coronary embolus from a degenerative calcific aortic valve-a rare cause of acute ST segment elevation myocardial infarction. Am Heart Hosp J. 2011;9(1):E55-E59.

29. Raphael CE, Heit JA, Reeder GS, et al. Coronary Embolus: An Underappreciated Cause of Acute Coronary Syndromes. JACC Cardiovasc Interv. 2018;11(2):172-180.

30. Johnson D, Gonzalez-Lavin L. Myocardial infarction secondary to calcific embolization: an unusual complication of bioprosthetic valve degeneration. Ann Thorac Surg. 1986;42(1):102-103.

31. Steiner I, Hlava A, Procházka J. Calcific coronary embolization associated with cardiac valve replacement. Necropsy x-ray study. Heart. 1976;38(8):816-820, 1.

32. James AH, Jamison MG, Biswas MS, Brancazio LR, Swamy GK, Myers ER. Acute myocardial infarction in pregnancy: a United States population-based study. Circulation. 2006;113(12):1564-1571.

33. Duarte FP, O'Neill P, Centeno MJ, Ribeiro I, Moreira J. Myocardial infarction in the 31 st week of pregnancy--case report [article in English, Portugese, Spanish]. Rev Bras Anestesiol. 2011;61(2):225-227, 228-231, 120-123.

34. Ladner HE, Danielsen B, Gilbert WM. Acute myocardial infarction in pregnancy and the puerperium: a populationbased study. Obstet Gynecol. 2005;105(3):480-484.

35. Abdel-Wahab M, Simonato M, Latib A, et al. Clinical valve thrombosis after transcatheter aortic valve-in-valve implantation. Circ Cardiovasc Interv. 2018;11(11):e006730.

\section{Author Affiliations}

Kelly Rasmussen, NP*; Roxann Rokey, MD ; Stacey C Rolak MPHt; Chuyang Zhong, MDई; John H Braxton, MD, MBA\|; Kazumasa Hashimoto, MD

*Cardiology Department, Marshfield Medical Center, Marshfield, WI USA

†Clinical Research Center, Marshfield Clinic Research Institute, Marshfield, WI USA

tUniversity of Wisconsin, School of Medicine and Public Health, Madison, WI USA

$\S$ Department of Internal Medicine, Marshfield Medical Center,

Marshfield, WI USA

"Department of Surgery, Billings Clinic, Billings, MT USA

\Obstetrics and Gynecology Department, Marshfield Medical

Center, Marshfield, WI USA 